\title{
Case series of trichotillomania presenting with three different DSM entities
}

\author{
Neha Modi ${ }^{1}$, Pradhyuman Chaudhary², Ritambhara Mehta ${ }^{1}$, Kamlesh Dave ${ }^{1}$ \\ ${ }^{1}$ Government Medical College, Surat, Gujarat, India \\ ${ }^{2}$ GMERS Medical College and Hospital, Sola, Ahmedabad, Gujarat, India \\ Neuropsychiatria i Neuropsychologia 2021; 16, 3-4: 196-199
}

Address for correspondence:

Pradhyuman Chaudhary, MD

GMERS Medical College, Sola, Ahmedabad

Gujarat, India

e-mail: drpradhyuman@gmail.com

\begin{abstract}
Trichotillomania (TTM; hair-pulling disorder) is classified as an obsessive-compulsive related condition within the Diagnostic and Statistical Manual of Mental Disorders, $5^{\text {th }}$ edition (DSM-5). Trichotillomania affects $4 \%$ of the population with significant comorbidity $(38.8 \%)$. It is found between trichotillomania and different DSM-5 entities such as schizophrenia, bipolar mood disorder or depressive disorders, obsessive-compulsive disorder, borderline personality disorder, obsessive-compulsive disorder (OCD) and with other body-focused repetitive behaviours (BFRBs) such as skin-picking disorder (SPD). We report three cases of trichotillomania with three different comorbid psychiatry disorders: schizophrenia, borderline personality disorder and bipolar mood disorder. Current treatment strategies for TTM involve a multimodal approach as according to research in the presence of comorbidity, treatment for TTM may need to be modified.
\end{abstract}

Key words: trichotillomania, treatment modality, psychiatric comorbidities.

\section{Introduction}

Trichotillomania (TTM; hair pulling disorder) is classified as an obsessive-compulsive related condition within the Diagnostic and Statistical Manual of Mental Disorders, $5^{\text {th }}$ edition (DSM-5) (APA, 2013). Trichotillomania affects $4 \%$ of the population (Kumar and Rajmohan 2012), with significant comorbidity (38.8\%) (Houghton et al. 2016) found between trichotillomania and different DSM-5 entities such as schizophrenia, bipolar mood disorder or depressive disorders, obsessive-compulsive disorder, borderline personality disorder, obsessive-compulsive disorder (OCD) and with other body-focused repetitive behaviours (BFRBs) such as skin-picking disorder (SPD) (Flessner et al. 2012; Houghton et al. 2016; Stein et al. 2008). Investigation of psychiatric comorbidity associated with TTM may assist in the identification of hair-pulling complexity. In clinical samples of patients with TTM, there are high rates of comorbid mood and anxiety disorders, and such comorbidities may be accompanied by more severe hair-pulling, greater problems with resistance and control over hair-pulling, poorer quality of life, and greater disability (Grant et al. 2017 a, 2017b). In this case series, our primary aim was to explore and investigate psychiatric comorbidities in TTM and their intervention.

\section{Case history}

We report three cases of TTM with three different comorbid psychiatric disorders: schizophrenia, borderline personality disorder and bipolar mood disorder.

\section{Case 1}

A 30-year-old female patient presented to the psychiatry outpatient department complaining of hearing of voices, persecutory ideas and fearfulness, suspiciousness, and pulling out her hair for the last 12 years and her psychotic symptoms had worsened in the last 2 months due to poor drug adherence along with urges of pulling scalp hair. She pulled hair from the scalp and developed patches in these areas. She had the irresistible desire to pull out her hair which she had expressed to her parents also. This act almost always occurred when she was alone, she had no work to do, she could not 
prevent herself from this act and if she tried to cut down, she felt restlessness which was relieved after pulling of hair. Hair pulling behaviour was not influenced by any psychotic features (delusion or commenting hallucination). In her past history she had hair pulling behaviour even with stable psychotic features. She had normal developmental milestones. Physical examination revealed an area of baldness over the scalp region (left and right parieto-temporal regions). It was a bizarre shaped patch of incomplete hair loss. The hairs on the patch were short, broken, and of uneven length. The hairs on the rest of the scalp were normal. A hair pull test was negative. Trichoscopy showed a sprinkled hair residue that is visible when hair shafts are almost totally damaged. On mental status she revealed auditory hallucinations of commentary type, persecutory ideas and urges for pulling hair. Her psychotic symptoms improved with $6 \mathrm{mg}$ of risperidone within two weeks, but her hair pulling behaviour improved only after adding $20 \mathrm{mg}$ of fluoxetine at the third week. She showed progressive improvement in the 2-month follow-up, with a noticeable increase in hair density.

\section{Case 2}

An 18-year-old female patient presented to the psychiatry outpatient department with anger outbursts and self-harm behaviour; she had adjustment problems with parents secondary to her socially unacceptable relationship with a male. She was apparently asymptomatic until 3 years previously, and she had started pulling her hair when she experienced extreme anxiety following conflict with her mother. Whenever she was alone, she pulled her scalp hair and hid this hair in her books and cupboards. She also had a complaint of an increasingly repetitive urge to pull out her hair, which gave her relief from her anxiety. Her parents knew about this habit when they found lots of hair-balls during cleaning of their house. Her parents noticed that she had loose hair on her scalp region and she used black colour or kajal to hide the areas of hair loss. She had consultations with many dermatologists and later psychiatrists, but she was not improving. She had normal developmental milestones. Physical examination revealed an area of baldness over the scalp region (frontoparietal region). Dermatological examination revealed diffuse thinning with short, jagged, and broken hairs, and negative hair pulling test. She revealed that during the last 3 years she had experienced easy irritability over par- ents secondary to not accepting her relationship with her male partner. On her mental status examination, she expressed feeling frustrated, lonely, anxious and depressed with labile affect. Thought was coherent and relevant. There were no delusions, obsessions or perceptual abnormality. She was conscious, well oriented to time, place and person, attention was easily aroused and sustained. Insight and judgement were fair. She also reported chronic mild suicidal ideation, explaining that it gave her relief to think about suicide as a way out from the current situation. However, she did not have any suicidal plan or attempt at present. At times, she thought that her parents were the best carers for her and at other times she thought that her parents did not understand her and ignored her. In her past history, she had non-suicidal self-harm behaviour 4 times, especially cutting her wrists, since she was a teenager. All incidents happened after quarrel with her friends or family members. The had a history of parental confrontation and wrangling in early childhood. She was very short tempered, demanding, reckless, irritable, occasionally aggressive. She had difficulty to control her anger and intense emotional fluctuation. She was diagnosed with borderline personality disorder with depression. Her depressive and TTM symptoms (reduced hair pulling behaviours and improved density) was improved gradually with $300 \mathrm{mg}$ of oxcarbazepine, $20 \mathrm{mg}$ of fluoxetine after 3 months. Prolonged psychological intervention was advised during the maintenance phase to learn how to interact with others and how to effectively express herself. Schema-focused therapy was chosen to target negative thoughts, feelings and behaviours. She showed progressive improvement in the 2-month follow-up, with a noticeable increase in hair density.

\section{Case 3}

A 40-year-old female patient presented to the psychiatry outpatient department with complaints of depressed mood without diurnal variation, feelings of worthlessness and hopelessness, sleep disturbance for 2 months along with scalp hair pulling behaviour. She began pulling at her scalp hair and it became a daily routine during the last 2 months. She started pulling her hair during times of stress, but only on occasions was aware that she was doing it. The rest of the time, she reported that she pulled "automatically" or without consciously noticing it and would notice a pile of hair on the floor or on her desk, before snapping out of it. She 
spent one to two hours each day on this hair pulling behaviour. The episode usually ended when the tugging sensation felt right and when she saw a good hair root on the pulled hair. The feelings of accomplishment from plucking quickly turned into shame and embarrassment. On her mental status examination, she reported that she felt worthless, had no motivation and lacked energy, did not feel like leaving the bed all day long. She felt the urge to move around restlessly. During this period she had hair pulling behaviour. Physical examination revealed an area of baldness over the scalp region (right parietotemporal regions). It was a bizarre shaped patch of incomplete hair loss. The hairs on the patch were short, broken, and of uneven length with a negative hair pull test. Trichoscopy showed broken hairs of uneven length, black dots, and a few yellow dots containing black dots. She had a past history of manic and depressive episodes without hair pulling behaviour. She was first diagnosed with depression 5 years ago and responded well to escitalopram $10 \mathrm{mg}$ once a day, which was discontinued after a year. Two years after the first episode she had mania and was treated with a mood stabiliser and anti-psychotic drug (details not available). After that she had 2 more manic and 1 depressive episode. She did not receive ECT in any episode. She did not have TTM in the past. She was improved with $1000 \mathrm{mg}$ of sodium valproate. She showed progressive improvement in the 6-month followup. Her hair pulling behaviour was improved along with depressive and anxiety symptoms.

\section{Discussion}

All three cases fulfil the criteria of TTM. Not all three cases had a history of hair ingestion after hair pulling behaviour. The course of all TTM symptoms was independent of co-morbid DSM-5 entities. According to patients, this hair pulling behaviour was not associated with symptoms of the co-morbid disorder. In the above TTM patients with schizophrenia and borderline personality disorder there was an improvement after addition of selective serotonin reuptake inhibitors (SSRIs) to atypical antipsychotics and a mood stabilizer, while the patient with bipolar mood disorder had improvement in TTM only with a mood stabilizer.

In clinical practice, potential presence of comorbidity must be assessed, which could affect treatment choice, response, and outcome. TTM is a disorder that results in loss of functionality in all aspects of life. In clinical samples from the literature, complex TTM cluster (63.16\%) showed comorbidity with major depressive disorder (62\%), panic disorder with/out agoraphobia $(8 \%)$, social anxiety disorder (15\%), specific phobia (22\%), generalized anxiety disorder $(22 \%)$, obsessive-compulsive disorder (36\%), skin-picking disorder (24\%), posttraumatic stress disorder $(15 \%)$, attention deficit disorder (8\%), tics (8\%), binge-eating disorder (6\%), body dysmorphic disorder (9\%), alcohol abuse and dependence (14\%), substance abuse and dependence (22\%). Also increased severity with comorbid TTM was noted (Lochner et al. 2019).

Current presence of depressive symptoms in patients with TTM is a predictor of quality of life (QOL) deficits and QOL may be improved by SSRIs (Houghton et al. 2016). In contrast, SSRIs are the most widely used treatment for both children and adults with TTM, despite evidence that their efficacy is no greater than placebo (Bloch et al. 2007). Researchers concluded that beginning with a thorough psychiatric assessment to establish an accurate diagnosis and assessing for co-occurring psychiatric disorders; medical evaluation for ingesting hair to assess for possible gastrointestinal blockage; providing education about the disorder to the patient, including aetiologies, treatment risks and benefits; providing habit reversal therapy (HBT) to treat TTM should be done (Grant et al. 2016). HBT is a cognitive behavioural therapy technique for TTM and Tourette's syndrome (Azrin et al. 1980). The HBT for TTM consists of four main components: self-monitoring; awareness training; stimulus control and stimulus-response intervention or competing response. The findings from a systematic review and meta-analysis demonstrate that HRT, when practised by experienced clinicians in academic research settings, is the most effective intervention for TTM. Furthermore, HBT demonstrated superiority in individual study trials when compared with the two most prevalent pharmacological interventions for trichotillomania: clomipramine and SSRI (on self-report but not blinded ratings of TTM severity). Also, clomipramine demonstrated efficacy for TTM when compared with placebo or active control conditions, while there was no evidence to suggest a benefit of SSRI compared with control conditions (Bloch et al. 2007).

In addition, the putamen is the key component in motor control and is involved in habit learning and response suppression across species (Morris et al. 2016). While the caudate plays a role in directed learning (such as during 
complex planning or high-level flexible learning tasks), the putamen is more involved in lowerlevel stimulus-response habit learning (Grahn et al. 2008). The macroscopic changes in putamen but not caudate volume associated with trichotillomania may help to account for why co-morbidity free patients with trichotillomania manifest response inhibition deficits (Odlaug et al. 2014), while other cognitive domains are relatively spared (Chamberlain et al. 2006). In conclusion, former researchers of clinical characteristics and comorbidity have significantly contributed to better understanding and more efficient treatment of this disorder. Several pathological mechanisms, including cognitiveaffective processes, neuropsychological deficits, and neurotransmitter systems, have been proposed. Individual structural brain abnormalities have been implicate for their role in co-morbid TTM patients irrespective of course of co-morbid DSM entity. In our cases, all three patients improved with pharmacological intervention, but psychological intervention was required in the patient with borderline personality disorder to treat personality-related problems and not for hair pulling behaviour. HBT is the preferred cognitive behavioural therapy technique for TTM and demonstrated superiority over clomipramine and SSRI, but SSRI improves QOL. Pharmacological intervention helps to treat TTM where HBT is not feasible due to acute comorbid psychiatric conditions. Additional pharmacological intervention is required to treat comorbid psychiatric conditions. In conclusion, current treatment strategies for TTM involve a multimodal approach as per research in the presence of co-morbidity, so treatment for TTM may need to be modified as per comorbidity.

\section{Declaration of patient consent}

The authors certify that they have obtained all appropriate patient consent forms. In the form the patient(s) has/have given his/her/their consent for his/her/their images and other clinical information to be reported in the journal. The patients understand that their names and initials will not be published and due efforts will be made to conceal their identity, but anonymity cannot be guaranteed.

\section{Disclosure}

The authors declare no conflict of interest.

\section{References}

1. American Psychiatric Association. Diagnostic and Statistical Manual of Mental Disorders, Fifth edition. American Psychiatric Association, Washington, DC 2013.

2. Azrin NH, Nunn RG, Frantz SE. Treatment of hair pulling (trichotillomania): A comparative study of habit reversal and negative practice training. J Behav Ther Exp Psychiatry 1980; 11: 13-20.

3. Bloch MH, Landeros-Weisenberger A, Dombrowski P, et al. Systematic review: pharmacological and behavioral treatment for trichotillomania. Biol Psychiatry 2007; 62: 839-846.

4. Chamberlain SR, Fineberg NA, Blackwell AD, et al. Motor inhibition and cognitive flexibility in obsessive-compulsive disorder and trichotillomania. Am J Psychiatry 2006; 163: 1282-1284.

5. Flessner CA, Knopik VS, McGeary J. Hair pulling disorder (trichotillomania): genes, neurobiology, and a model for understanding impulsivity and compulsivity. Psychiatry Res 2012; 199: 151-158.

6. Grahn JA, Parkinson JA, Owen AM. The cognitive functions of the caudate nucleus. Prog Neurobiol 2008; 86: 141-155.

7. Grant JE, Chamberlain SR. Trichotillomania. Am J Psychiatry 2016; 173: 868-874.

8. Grant JE, Redden SA, Leppink EW, Chamberlain SR. Trichotillomania and co-occurring anxiety. Compr Psychiatry 2017a; 72: 1-5.

9. Grant JE, Redden SA, Medeiros GC, et al. Trichotillomania and its clinical relationship to depression and anxiety. Int J Psychiatry Clin Pract 2017b; 21: 302-306.

10. Houghton DC, Maas J, Twohig MP, et al. Comorbidity and quality of life in adults with hair pulling disorder. Psychiatry Res 2016; 239: 12-19.

11. Kumar PN, Rajmohan V. Trichotillomania with trichorhizophagia in a schizophrenic patient: case report and review of literature. Indian J Psychiatry 2012; 54: 196197.

12. Lochner C, Keuthen NJ, Curley EE, et al. Comorbidity in trichotillomania (hair-pulling disorder): A cluster analytical approach. Brain Behav 2019; 9: e01456.

13. Morris LS, Kundu P, Dowell N, et al. Fronto-striatal organization: Defining functional and microstructural substrates of behavioural flexibility. Cortex 2016; 74: 118-133.

14. Odlaug BL, Chamberlain SR, Derbyshire KL, et al. Impaired response inhibition and excess cortical thickness as candidate endophenotypes for trichotillomania. J Psychiatr Res 2014; 59: 167-173.

15. Stein DJ, Flessner CA, Franklin M, et al. Is trichotillomania a stereotypic movement disorder? An analysis of body-focused repetitive behaviors in people with hair-pulling. Ann Clin Psychiatry 2008; 20: 194-198. 